\title{
Anaesthesia Recommendations for Patients with Loeys-Dietz Syndrome
}

\author{
Aysun Postaci and İsmail Aytaç* \\ Department of Anesthesiology and Reanimation, Ankara Numune Education and Research Hospital, Turkey
}

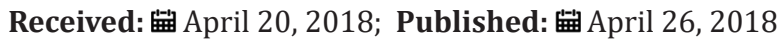

*Corresponding author: İsmail Aytac, Department of Anesthesiology and Reanimation, Ankara Numune Education and Research Hospital, Turkey

\author{
Abstract \\ Loeys-Dietz Syndrome is a otosomal dominant connective tissue disorder which has characteristic triad with \\ a) Arterial tortuosity, aneurysms or dissections; \\ b) Hypertelorism; and \\ c) Bifid uvula or cleft palate.
}

This review summarizes the features of anesthesia management in this syndrome

Keywords: Loeys-Dietz Syndrome; Anesthesia management

Abbreviations: LDS: Loeys-Dietz Syndrome; TGFBR1: Transforming Growth Factor-Receptor Type 1; ARB: Angiotension Receptor Blockers; ACE: Angiotension Converting Enzyme

\section{Introduction}

Loeys-Dietz Syndrome (LDS), is a rare connective tissue disease which is classified under Marfan-like dysfunctions and described by Loeys et al. [1]. LDS is inherited in an otosomal dominant manner. Four types of LDS are described according to mutations in genes which play important roles in cell signalling that promotes production of proteins for tissue growth and development. While the cytokine transforming growth factor-receptor type 1(TGFBR1) gene mutations cause type I and the TGFBR2 gene mutations cause type II, mutations in the SMAD3 and TGBF2 genes result in type III and type IV, respectively. The most common forms of disease are types I and II [2]. The prevelance of this syndrome is less than $1 / 1$ 000000 [3]. The diagnostic triad of this syndrome is as follows:
a) Arterial tortuosity, aneurysms or dissections;
b) Hypertelorism; and
c) Bifid uvula or cleft palate.

Although, arterial tortuosity is observed mostly in the head and neck vessels, it can occur other vessels as well. The clinical findings

for musculoskelatal system involvement can be arachnodactyly, joint laxity, pectus deformities, scoliosis, dolichosternomelia, talipes equinovarus, comptodactyly, and cervical spine instability. Cardiovascular findings can be aortic diltation and disection, bicuspid aortic valve, and congenital heart disease such as atrial septal defect, patent ductus arteriosis. Central nervous system anomalies such as Chiari malformation and hydrocephalus can be observed. However, the life treatining complications that determine the prognosis are mostly cardiovascular system related findings like aortic root aneurysms, arterial tortuosity, aneurysyms of other vessels, patent ductus arteriosis, and atrial septal defect.

\section{Frequently Performed Surgery Types}

Patients can have surgeries (emegency or elective) under anesthesia for cardiovascular (aortic aneurysm repairment, aortic valve replasman), orthopeadic (musculoskeletal system anomalie repairment), neurosurgical (for vascular, craniosynostosis, chari malformation and hydrocephalus), ophtalmic (for cataracts, strabismus, amblyopia, and exotropia), and general (for inguinal, umblical and hiatal hernia repairment, splenic or bowel rupture). 


\section{Type of Anesthesia}

Publications about LDS in literature are usually about surgical treatments of anomalies and associated symptoms. Anesthesia for patients with LDS should be special. Anesthetic tecnique should be decided according to the pathologies that patients have by an experienced anesthesiologist. Aorta and other big vessels as well as all cardiovascular system are affected by hypotension or hypertension during anesthesia because of lack of elasticity in the vessels. Anesthetic approach for Marphan syndrome can be applied for LDS patients since it is described as Marphan-like syndrome.

\section{Additional Necessary Preoperative Diagnostic Proce- dures}

First, it should be investigated if there are cardiovascular anomalies in children since these anomalies usually asymptomatic in earlier ages. Diagnostic tests such as electrocardiogram, echocardiogram, or cardiac magnetic resonance imaging should be ordered preoperatively to rule out cardiac or aortic pathology. In case of aneurysms, it should be consulted with neurovascular and cardiovascular surgeons for surgical plan. Although, the risk of aortic dissection increases at or above the $5.0 \mathrm{~cm}$ aortic root dimension in Marphan syndrome, dissections have been reported in patients with LDS types I, II, or III when aortic root dimensions are 3.9, 4.0, 1.3 $\mathrm{cm}$, respectively. Also, dissections have been reported in LDS type IV patients with aortic root dimension under $5.0 \mathrm{~cm}$ [4-6]. When the maximal aortic root dimension reaches $4.0 \mathrm{~cm}$ in in adult patients with LDS type I or II, surgery is performed, however, in children surgery can be postponed until the aortic root reaches $2.0-2.2 \mathrm{~cm}$ to accommodate adult-sized graft. Children with mild craniofacial features and slowly progressive aortic root dilatation, surgery can be delayed until aortic root dimension is $4.0 \mathrm{~cm}$, however, children with severe craniofacial features, rapidly progressive aortic dilatation $(0.5 \mathrm{~cm}$ over a year), as well as family history of agressive aortic disease earlier surgery should be considered.

To asses the instability of cervical spine, X-rays at flexion and extension positions should be performed. In the presence of spontaneous pneumothoraces history, chest X-ray also should be ordered. Pulmonary function tests should be done in patients with severe scoliosis to evaluate the extent of restrictive lung disease. Ophtalmic examination and required treatments should be performed. These patients are prone to have high prevelance of immunologic features, so for this reason allergy history should be taken carefully.

\section{Particular Preparation for Airway Management}

Airway management might be hard due to craniofacial deformities, cleft palate, micrognathia, retrognathia, cervical spine abnormalities, subluxations, or instability. Difficulties of anesthesia should be explained to patients and their relatives and consent form asked to be signed. Difficult entubation and airway management table should be ready. During preoperative examination for airway evaluation, deformities, situation of teeth, as well as opening of nostrils should be recorded. Mallampathy clasification, evaluates degree of apparence of palates in adults, can not be used for babies and young children. It can only be used in older children who can apply commands [7]. Entubation using videolaryngoskopy can be preferred in these patients due to difficult airway management and cervical spine instabilities [8]. Anesthesia should be planned according to the area of surgery, duration of surgery, and degree of invasivity. Also, an alternative plan should be thought. Airway masks may not be appropriate and ventilation might be difficult. Airway obstruction might be observed due to structural deformities like cleft palate [9]. Laryngeal masks can be used successfully if general anesthesia is not required entubation and the duration of anesthesia is less than two hours. Hypertensive response to entubation and risk of aortic dissection would be eliminated using laryngeal masks. If endotracheal entubation will be applied, to prevent hemodinamic changes due to entubation precautions should be prepared. In the presence of pneumothoraces and restrictive lung disease, lung protective strategies should be applied and use of N2O should be avoided.

\section{Preparations for Transfusion or Administration of Blood Products}

There is no evidence that shows that LDS patients with preexisting coagulation abnormalities have a higher risk of blood transfusion.

\section{Preparations for Anticoagulan Treatment}

There is no information suggesting anticoagulan treatment except for patients who underwent valve or aortic root replacement surgery. Patients required anticoagulation due to prosthetic valve should be offered a bridging therapy prior to elective surgery. Bridging therapy is not offered to patients newly started oral anticoagulan treatment for atrial fibrillation because of its quick and short duration of effect $[10,11]$.

\section{Precautions for positioning, transportation, and/or mo- bilization}

To prevent joint injury and dislocatins due to musculoskeletal system deformities, patients should be positioned appropriately and areas stay under pressure should be supported using soft position pillows. Especially in prone position, head should be hold in neutral position and excessive rotations should be avoided because of possible cervica spine instability.

\section{Possible İntereactions between Anesthetic Agents and Patients' Long Term Medications}

Anti-hypertensive medications (angiotension receptor blockers (ARB), $\beta$-blockers, and angiotension-converting enzyme (ACE) inhibitors): $\beta$-blockers should be continued perioperatively. Side effects of perioperative $\beta$-blockage are bradycardia and hypotension. To continue ACE inhibitors and ARBs until surgery 
may increase the risk of perioperative hypotension, however, it should be kept in mind that cease of ACE inhibitors prior to surgery may lead postoperative hypertension [12].

\section{Anesthesia}

Anesthesia induction requires a special care in these patients. Severe hypotension or hypertension should be avoided. There is not any described method for choosing anesthetic agent [13] Anesthesia induction via intravenous route can cause hypoxia following sudden lost of airway management and apnea in patients with difficult airway. However, anesthesia induction via inhalation gives a chance to control airway and continue of spontaneous breathing. Emergency cardiac medications should be ready to treat radically in case of development of critical situations like hypotension and/or rhythm problems. As a vasopressor phenylephrine should be preferred over ephedrine that may induce tachycardia through its beta-adrenergic effect. Central lines should be placed using ultrasound because of cervicovertebral arterial tortuosity by an experienced anesthetic team.

Antibiotic proflaxia for high risk procedures should be thought in patients who have prosthetic valve or have prosthetic material for valve repairment; had endocarditis previously; or have congenital heart disease [14]. High risk procedures that suggested endocarditis proflaxia are follows: dental procedures (only gingival or periapical region manuplations, oral mucosa perforations), airway procedures (in case of infection, during bronchoscopy, laryngoscopy, transnasal, or endotracheal entubation), gastrointestinal and urogenital procedures (in existence of infection, gastroscopy, colonoscopy, cystoscopy, and transeusophageal echocardiography), skin and soft tissue procedures (if there is no infection, no need any antibiotic prophylaxia) [15].

\section{Monitoring}

Additional to routine anesthetic monitoring (ECG, non-inasive blood pressure, Sp02, end-tidal C02) airway pressures, anesthetic gases, core temmperature, and neuromuscular blockage should be monitored. Invasive-blood pressure monitoring should be used intraoperatively to notice sudden changes.

\section{Possible Complications}

Complications of LDS are secondary to conditions, symptoms, or diseases that are involved in LDS. There are rupture of aortic aneurysm, cerebral hemorrhage, cardiac arrhythrmias, rupture of spleen or bowel, pneumothoraces, aortic dissections during pregnancy or immediate postpartum, and rupture of uterine.

\section{Postoperative Care}

Postoperative monitoring should be decided according to type of surgical procedure, problems developed intraoperativly, ventricular function, as well as condition of aortic root. An intense follow up might be needed for obstructive sleep apnea syndrome in children with craniofacial abnormalities. These patients can be monitored postoperatively in a first step intensive care unit. Kuisle et al. [16] reported an adolescent patient with LDS and undiagnosed adrenoleukodystrophy who developed adrenal crisis. To prevent excessive endogeneous cathecholamine production, it is essential to control pain and anxiety in these patients.

\section{Ambulatory Anesthesia}

There are no reported experiences of patients with LDS in the ambulatory setting.

\section{Obstetrical Anesthesia}

Although women with LDS tolerated pregnancy well, vaginal delivery, or cessarian section with close follow up and early action was still required, and were accepted as high risk patients. Early delivery and avoiding of intra- abdominal pressure increase while applying cessarian section may decrease obstetric complications risk. As of today, there has been three pregnant LDS patients reported. The first case was a 16 weeks pregnant LDS patient who had successful surgical aortic valve and arch replacement for type A aortic dissection. She had an uneventful cessarian delivery [17]. The second case was an LDS patient who underwent cessarian section without any complications at 34 weeks pregnancy. There was no information about anesthesia for these patients. The third case was an LDS patient 36 weeks pregnant with $3.45 \mathrm{~cm}$ aortic root dilatation who underwent c- section under general anesthesia without any complications [18].

LDS was described as Morphan-like syndrome in 2005 and anesthesia management can be adopted from anesthesia application for patients with Morphan syndrome until described. Higher complication risks including death from aortic dissection was reported in pregnant women with aortic dimension over $4.5 \mathrm{~cm}$ in Morphan syndrome [19]. Since LDS is more severe, higher risk of vascular complications can be expected. In female patients who have around $4.0 \mathrm{~cm}$ aortic root, aortic root replacement should be considered before pregnancy. Whole aorta should be evaluated before pregnancy. If needed, surgical aortic root dilatation can be applied before 28 weeks of pregnancy [20].

\section{Management of Neuraxial Analgesia or Anesthesia}

Neuraxial anesthesia might bring some technical difficulties due to scoliosis. Also, dural ectasia which is widening or balloning of the dural sac around the spinal cord might be encountered in these patients. Dural estesia develops due to structural changes of elastin. This might lead to dural puncture during epidural catheter placement or unsucessful spinal anesthesia [21,22]. For this reason, ultrasonographic evaluation of lumbar spinal area is suggested before neuroanesthesia [23]. Continue spino- epidural anesthesia can be preferred in these patients. This gives an opportunity to complete to the cessarian section by epidural anesthesia after an unsuccessful spinal anesthesia as well as to achieve post- operative pain management. 


\section{Management of General Anesthesia}

Hemodynamic responses to laryngoscopy and surgery are important for LDS patients with dilatated aortic root during general anestesia. Hemodynamic responses to laryngoscopy and surgery can be prevented by continuing beta- blocker treatment and by administering an iv opioid during the induction of anesthesia. Ultra- short acting remifentanyl which has no important effects in newborns can be preferred. Remifentanyl 0.5-1mcg/ $\mathrm{kg}$ bolus administration effectively reduces hemodynamic and cathecolamine release responses in pre eclampsia, but still it should be kept in mind that it can cause temporary respiratory depression in newborns [24,25].

Table 1: Anesthestic preparations for patients with LDS.
There is no evidence that one anesthestic technique is superior to another one [22]. It is more essential to avoid hypertension rather than mild hypotension. For this reason, one might said that, this problem can be attenuated using neuraxial anesthesia instead of general anesthesia. To determine sudden arterial pressure changes in pregnant LDS patients, invasive arterial pressure monitoring is required. Even, transesophageal echocardiogram can be suggested. Also, postoperative pain management should be planned. It is suggested that these patients should be cared in intensive care unit for postoperative $24 \mathrm{hrs}$ for possible aortic arterial or hollow organ ruptures.

\begin{tabular}{|c|c|}
\hline \multirow{10}{*}{$\begin{array}{l}\text { Preoperative } \\
\text { evulation }\end{array}$} & a) Cervical spine X-rays in flexion and extension position performed at diagnosis to assess for instability \\
\hline & b) Full vascular imaging prior to pursuing pregnancy \\
\hline & c) Consultation with cardiovascular/neurovascular specialists for surveillance and/or surgical plan with presence of aneuryms \\
\hline & d) Pulmonary consultation or sleep study for sleep apnea in presence of clinical symptoms \\
\hline & e) History of allegry should be carefully taken. Children with frequent surgical procedures may have developed Latex allergy. \\
\hline & f) Check for airway difficulties \\
\hline & $\begin{array}{l}\text { g) Inform the patients or relatives about relevant issues of the underlying disorder with regard to planned anestesia. Discuss specific } \\
\text { anesthetic risk of general or neuroaxial anesthesia. }\end{array}$ \\
\hline & h) If needed, endocarditis prophylaxis should be administered. \\
\hline & i) If needed, deep vein thrumbosis prophylaxis should be administered. \\
\hline & j) Beta- Blocker treatment should be continued. Patients treated with ACE- I and ARBs should be carefully observed for hypotension. \\
\hline \multirow{8}{*}{$\begin{array}{l}\text { Anesthesia } \\
\text { considerations }\end{array}$} & a) Airway management table and alternative plans should be prepared for patients expected to have difficult airways. \\
\hline & b) Invasive arterial pressure monitoring should be applied. \\
\hline & c) A smooth anesthesia induction should be performed by avoiding hypo- hypertension. \\
\hline & d) Avoid peroperative hypertension and apply careful hemodynamic monitoring during surgery. \\
\hline & e) During central catheterization, use ultrasonography. \\
\hline & f) During neuraxial anesthesia, use ultrasonography to assess possible dural ectesia \\
\hline & g) During positioning, areas under pressure should be supported using position pillows. \\
\hline & h) During extubation, avoid hypertension. \\
\hline \multirow{3}{*}{$\begin{array}{l}\text { Postoperative } \\
\text { Care }\end{array}$} & a) Postoperative acute pain management should be planned. \\
\hline & b) Prevent hypertensive attacks. \\
\hline & c) 24 hour intensive care unit monitorization. \\
\hline
\end{tabular}

\section{Conclusion}

Although LDS is a rare disease, it should be considered in terms of anesthesia as it may require early and repeated surgical intervention; also has many medical features that threaten life.

\section{References}

1. Loeys BL, Chen J, Neptune ER (2005) A syndrome of altered cardiovascular, craniofacial, neurocognitive and skeletal development caused by mutations in TGFBR1 or TGFBR2. Nat Genet 37(3): 275-281.

2. El Hamamsy I, Frischmeyer Guerrerio PA, MacCarrick G, Black III JH, Bowdin S, et al. (2014) Loeys-Dietz syndrome: A primer for diagnosis and management. Genet Med 16(8): 576-587.

3. Bunting AC, Bould D (2014) Hemodynamic instability during anesthesia in an adolescent with Loeys-Dietz syndrome: A case report. Paediatr Anaesth 24(12): 1302-1304.
4. Loeys BL, Schwarze U, Holm T (2006) Aneurysm syndromes caused by mutations in the TGF-beta receptor. N Engl J Med 355(8): 788-798.

5. Mirghassemi A, Soltani AE, Abtahi M (2011) Evaluation of laryngoscopic views and related influencing factors in a pediatric population. Pediatric Anaesth 21(6): 663-667.

6. (2010)ACCF/AHA/AATS/ACR/ASA/SCA/SCAI/SIR/STS/SVM Guidelines for the Diagnosis and Management of Patients with Thoracic Aortic Disease. Circulation 121(13): e266-e369.

7. Crocker K, Black AE (2009) Assessment and management of predicted difficult airway in babies and children. Anaesth Int Care Med 10: 200205.

8. Postaci A, Tiryaki C, Aytac I, Dikmen B (2012) Anesthetic management of a child with Loeys-Dietz Syndrome-case report. Journal of Anesthesia (Anestezi Dergisi) 20(2): 114-118.

9. Butler Mg, Hayes BG, Hathaway MM (2000) Specific genetic diseases at 
risk for sedation/anesthesia complications. Anesth Analg 91(4): 837 855.

10. (2014) ESC/ESA Guidelines on non-cardiac surgery:cardiovascular assessment and management. European Heart Journal 35: 2383-2431.

11. Bunting AC, Bould DA (2014) Hemodynamic instability during anestesia in an adolescent with Loeys-Dietz syndrome: A case report. Pediatric Anesth 24(12): 1302-1303.

12. Pigott DW, Nagle C, Allman K, Westaby S, Evans RD (1999) Effect of omitting regular ACE inhibitor medication before cardiac surgery on haemodynamic variables and vasoactive drug requirements. $\mathrm{Br} \mathrm{J}$ Anaesth 83(5): 715-720.

13. Norris EJ (2010) Anesthesia for vascular Surgery. In: Miller RD ( $7^{\text {th }}$ Edn); Miller's Anesthesia. Churchill Livingstone Elsevier 2: 2011.

14. (2012) Guidelines on the management of valvular heart disease. European Heart Journal 33(19): 2451-2496.

15. (2015) ESC Guidelines for the management of infective endocarditis. European Heart Journal 36(44): 3075-3123.

16. Kuisle AM, Gauguet S, Karlin LI, Dauber A, McCann ME (2011) Postoperative adrenal crisis in an adolescent with Loeys-Dietz syndrome and undiagnosed adrenoleukodystrophy. Can J Anesth 58(4): 392-395.

17. Kunishige H, Ishibashi $Y$, Kawasaki M, Yamakawa T, Morimoto K, et al (2012) Surgical treatment for acute type A aortic dissection during pregnancy (16 weeks) with Loeys-Dietz syndrome. Gen Thorac Cardiovasc Surg 60(11): 764-767.

18. Cronin J, Cuschieri HB, Dong X, Oswald G, Russo M, et al. (2015) Anesthesia Considerations for Cesarean Delivery in a Patient with Loeys-Dietz Syndrome. A\&A Case Reports 4(4): 47-48.
19. Harris IS (2011) Management of pregnancy in patients with congenital heart disease. Prog Cardiovasc Dis 53(4): 305-311.

20. Wu P, Poole TC, Pickett Ja, Bhat A, Lees CC (2013) Current Obstetric guidelines on tromboprophylaxis in the Unite Kingdom: Evidence based medicine? European Journal of Obstetrics \& Gynecology and Reproductive Biology 168(1): 7-11.

21. Lacassie HJ, Millar S, Leithe LG, Muir HA, Montaña R, et al. (2005) Dural ectasia: A likely cause of inadequate spinal anaesthesia in two parturients with Marfan's syndrome. Br J Anaesth 94(4): 500-504.

22. Allyn J, Guglielminotti J, Omnes S, Guezouli L, Egan M, et al. (2013) Marfan's Syndrome During Pregnancy: Anesthetic Management of Delivery in 16 Consecutive Patients. Anesth Analg 116(2): 392-398.

23. Chin KJ, Karmakar MK, Peng P (2011) Ultrasonography of the adult thoracic and lumbar spine for central neuraxial blockade. Anesthesiology 114(6): 1459-1485.

24. Yook KY, Kang DH, Jeong H, Jeong CW, Choi YY, et al. (2013) A doseresponse study of the hypertensive response to layngoscopy and tracheal intubation in severly preeclemptic women undergoing caesarean delivery under general anaesthesia. Int J Obstet Anesth 22(1): 10-18.

25. Park BY, Jeong CW, Jang EA, Kim SJ, Jeong ST, et al. (2011) Doserelated attenuation of cardiovascular responses to tracheal intubation by intravenous remifentanil bolus in severe pre-eclemptic patients undergoing Ceasarean delivery. Br J Anaesth 106(1): 82-87.

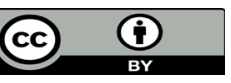

This work is licensed under Creative Commons Attribution 4.0 License

To Submit Your Article Click Here: Submit Article

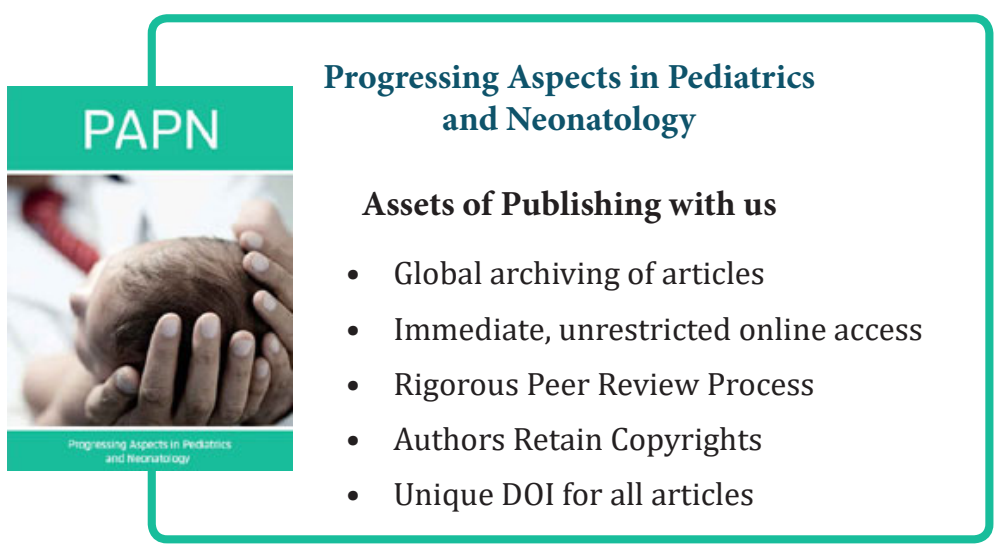

